\title{
Research Paper \\ Prevalence of Non-Communicable Disease Risk Factors Among the Elderly of Birjand in 2014
}

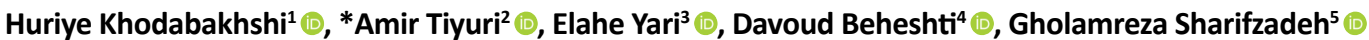

1. Department of Public Health, School of Public Health, Birjand University of Medical Sciences, Birjand, Iran.

2. Department of Epidemiology, School of Public Health, Iran University of Medical Sciences, Tehran, Iran.

3. Cardiovascular Diseases Research Center, Birjand University of Medical Sciences, Birjand, Iran.

4. Khaf Municipality Health Services Center, Mashhad University of Medical Sciences, Khaf, Iran

5. Social Determinants of Health Research Center, Birjand University of Medical Sciences, Birjand, Iran.

Received: 23 Aug 2018

Accepted: 09 Jan 2019

Available Online: 01 Apr 2019

Key words:

Prevalence, Non-

communicable

disease, Risk factor

\section{A B STRACT}

Objectives Noncommunicable diseases are the leading causes of death and disability, globally. Moreover, the aging population is growing in the world. These issues are among major public health challenges; thus, this study aimed to determine the prevalence of noncommunicable diseases risks factors among the elderly of Birjand City, Iran, in 2014.

Methods \& Materials The present cross-sectional study was conducted among 400 elderly who were selected through random cluster sampling method. Data collection was carried out using the World Health Organization STEPwise approach to noncommunicable diseases surveillance (STEPS) instrument. Furthermore, physical assessments included measuring height, weight, waist and hip circumference and blood pressure by trained health experts. Then, the obtained data were analyzed by Chi-squared test, t-test and one-way Analysis of Variance (ANOVA).

Results From 400 elderly who participated in this study, 200 persons were male and the Mean \pm SD age of study participants was $70 \pm 6.4$ years. The prevalence of smoking was $10.7 \% ; 49 \%$ were overweight or obese, and $34.5 \%$ had hypertension. The prevalence of abdominal obesity and physical inactivity were $63.5 \%$ and $55 \%$, respectively. In addition, the inadequate intake of fruits, vegetables, fish and dairy products were $73.8 \%$, $67.8 \%, 95 \%$, and $74.5 \%$, respectively. The prevalence of smoking in men, and the prevalence of obesity and overweight, physical inactivity and abdominal obesity in women were significantly higher $(P<0.01)$. However, there was no significant gender difference in the prevalence of hypertension ( $P=0.14)$.

Conclusion The achieved results indicated a high prevalence of noncommunicable diseases risks factors among the studied elderly. Given the importance of the growing elderly population, planning for appropriate interventions aimed for healthier, longer and higher quality lives in the elderly should be considered by health officials and policymakers.

\section{* Corresponding Author:}

Amir Tiyuri, PhD.

Address: Department of Epidemiology, School of Public Health, Iran University of Medical Sciences, Tehran, Iran.

Tel: +98 (936) 8896017

E-mail: amir.turi@yahoo.com 


\section{Extended Abstract}

\section{Introduction}

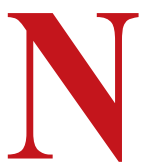

on Communicable Diseases (NCDs) are a global health problem and a threat to the health and development of the countries $[1,2]$. Elevating life expectancy and rapid population growth have increased the burden of NCDs [2, 4-9]. Such diseases are estimated to be responsible for $73 \%$ of all deaths and $60 \%$ of the disease burden in 2020 [8]. Major risk factors for NCDs in most countries include hypertension, diabetes, obesity, high blood lipids, smoking, inappropriate nutrition and physical inactivity $[2,4,5$, $7,10-13]$. The growing population of elderly in Iran is estimated to make up $10 \%$ of its population by 2021 [17]. In addition, considering the importance of NCDs among older adults, this study aimed to investigate the prevalence of risk factors for noncommunicable diseases among older adults in Iran. We attempted to provide a basis for future interventions on reducing the risk factors for such diseases in the elderly.

\section{Participants and methods}

This analytical correlational study with a crosssectional design was conducted in 2014. The study population consisted of all the elderly in Birjand City, Iran. Of them, 400 were selected as study samples by random cluster sampling method. First, Birjand City was divided into 4 regions based on geographical status. Then, 40 cluster heads were assigned based on the random postal codes received from the post office (10 clusters/4 regions).

Each cluster head comprised the postal address of a house. Sampling in each cluster head was started from the assigned house; and by moving to the right direction from the assigned house, 10 older adults aged $\geq 60$ years ( 5 men and 5 women) were selected. Data were collected by trained health professionals and through interviews and using the World Health Organization STEP wise approach to noncommunicable diseases surveillance (STEPS) instrument. The first steps consisted of exploring demographic information and measuring tobacco use, diet, and physical activity; and the next step comprised physical measurements (i.e. blood pressure, height, weight, waist, and hip circumference). In this regard, a portable digital scale (SEKA model) and a portable measurement rod for measuring the weight and height of samples; a flexible nonstretching measuring tape for assessing waist and hip circumference; and a sphygmomanometer (Riester, Germany) for measuring blood pressure were used. Eventually, the collected data were analyzed in SPSS by descriptive statistics and Chi-squared test, Independent Samples t-test, and one-way Analysis of Variance (ANOVA). The significance level was set at $\mathrm{P}=0.05$. This study was approved by the Research Ethics Committee of Birjand University of Medical Sciences (code: IR.BUMS.1394.82).

\section{Results}

Of 400 participants, $200(50 \%)$ were male with the Mean \pm SD age of $70 \pm 6.4$ years. Moreover, $177(44.2 \%)$ were illiterate; $169(42.3 \%)$ reported a junior high school education, and 54(13.5\%) senior high school and above. In total, $3(0.7 \%)$ were single; $301(75.2 \%)$ married; 73(18.3\%) widow/widower, and 23(5.8\%) divorced. A total of $43.3 \%$ reported the consumption of liquid vegetable oil; $41 \%$ solid vegetable oil; $12.8 \%$ butter or animal oil, and $2.9 \%$ consumed other oil types. Moreover, $64.5 \%$ of men and $35.5 \%$ of women

Table 1. The gender-wise prevalence

\begin{tabular}{|c|c|c|c|c|c|c|}
\hline \multirow{2}{*}{ Prevalence } & Tobacco Use & $\begin{array}{l}\text { Hookah } \\
\text { Use }\end{array}$ & $\begin{array}{l}\text { Overweight and } \\
\text { Obesity }\end{array}$ & $\begin{array}{l}\text { Abdominal } \\
\text { Obesity }\end{array}$ & $\begin{array}{l}\text { High Blood Pres- } \\
\text { sure }\end{array}$ & $\begin{array}{l}\text { Physical Inactiv- } \\
\text { ity }\end{array}$ \\
\hline & \multicolumn{6}{|c|}{ No. (\%) } \\
\hline \multirow{2}{*}{ Gender } & $32(16)$ & $21(10.5)$ & $74(37)$ & $93(46.5)$ & $76(38)$ & $96(48)$ \\
\hline & $11(5.5)$ & $9(4.5)$ & $122(61)$ & $161(80.5)$ & $62(31)$ & $124(62)$ \\
\hline$P$ & 0.001 & 0.023 & $<0.001$ & $<0.001$ & 0.141 & 0.005 \\
\hline Total & $43(10.7)$ & $30(7.5)$ & $196(49)$ & $254(63.5)$ & $138(34.5)$ & $220(55)$ \\
\hline
\end{tabular}


added table salt to food, and the difference between them was significant $(\mathrm{P}<0.001)$.

The prevalence rate of smoking was $10.7 \%$ and men were significantly more likely to use tobacco $(\mathrm{P}=0.001)$. The prevalence rate of hookah smoking was $7.5 \%$, which was significantly higher in men $(\mathrm{P}=0.023)$. The Mean \pm SD Body Mass Index (BMI) score of samples was $26.4 \pm 5.2 \mathrm{~kg} / \mathrm{m} 2$ which was significantly higher in women $(\mathrm{P}<0.001)$. In this regard, 19\% were lean, $32 \%$ normal, $25.8 \%$ were overweight, and $23.2 \%$ were obese; the prevalence of overweight and obesity was significantly higher in women $(\mathrm{P}<0.001)$. In respect of the waist-hip ratio, $63.5 \%$ of the samples had abdominal obesity; its prevalence was significantly higher in women $(\mathrm{P}<0.001)$. The Mean $\pm \mathrm{SD}$ systolic and diastolic blood pressure scores of samples were 124.4 \pm 13.5 and $83.6 \pm 11.9 \mathrm{mmHg}$, respectively. The prevalence of hypertension among subjects was $34.5 \%$, with no significant gender difference $(\mathrm{P}=0.14)$. A total of $39 \%$ of samples had not measured their blood pressure over the past year. In terms of diet, the Mean \pm SD fruit, vegetable, and fish consumption of samples were 4.1 \pm 1.8 , $3.3 \pm 2$, and $0.5 \pm 0.6 \mathrm{~d} / \mathrm{wk}$, respectively. Furthermore, their Mean \pm SD dairy consumption was $4.2 \pm 1.8 \mathrm{~d} /$ wk; the Mean \pm SD consumption of carbonated soft drinks was $1.3 \pm 1.4 \mathrm{~d} / \mathrm{wk}$; and the Mean $\pm \mathrm{SD}$ fast food consumption was $0.0 \pm 5.8 \mathrm{~d} / \mathrm{wk}$. Moreover, the inadequate consumption rate of fruits, vegetables, fish and dairy products was $73.8 \%, 67.8 \%, 95 \%$, and $74.5 \%$, respectively. In terms of physical activity, 55\% failed to report a minimum 10-min/d walking, and the prevalence of physical inactivity was significantly higher in women $(\mathrm{P}=0.005)$ (Table 1).

\section{Conclusion}

The obtained results indicated the significant and high prevalence of NCD risk factors, especially overweight and obesity, physical inactivity, abdominal obesity, and inappropriate nutrition in the studied older adults. The NCDs are the leading cause of mortality and disability in the world, and aged population and their growth are important. Thus, planning for comprehensive educational interventions for smoking cessation, creating dietary diversity and balance, performing age-appropriate exercises, and improving lifestyle with the goal of preventing NCDs and having a healthier, longer, and more quality life in the elderly should be addressed by health officials and policymakers.

\section{Ethical Considerations}

Compliance with ethical guidelines

This study was approved by the Ethics Committee of Birjand University of Medical Sciences with the code of IR.BUMS.1394.82.

\section{Funding}

This research has been extracted from the research project (No. 58.92) funded by Birjand University of Medical Sciences.

\section{Authors contributions}

Conceptualization: Huriye Khodabakhshi, Gholamreza Sharifzadeh; Methodology: Huriye Khodabakhshi, Amir Tiyuri; Analysis: Amir Tiyuri; Research and review: Elahe Yari, Davoud Beheshti; Writing-review and editing: All authors.

\section{Conflict of interest}

The authors declared no conflict of interest.

Acknowledgements

We thank the Vice-president of Research and Technology of Birjand University of Medical Sciences. 


\title{
شيوع عوامل خطر بيمارىهاى غيرواتير در سالمندان شهر بيرجند در سال سوسا
}

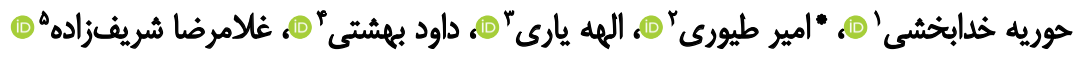 \\ 1 - كروه بهداشت عمومى، دانشكده بهداشت، دانشكاه علوم يزشكى بير جئده بيرجئده ايران.

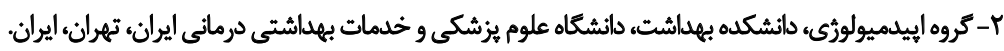

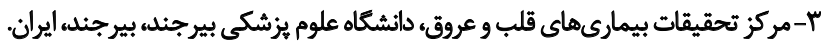

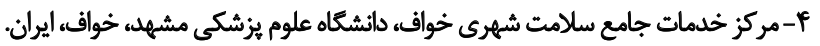

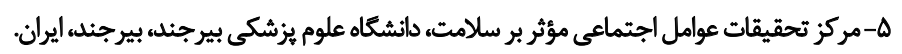

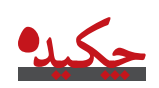

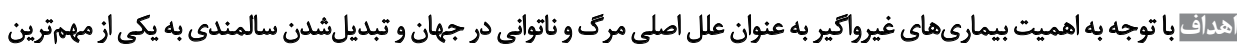

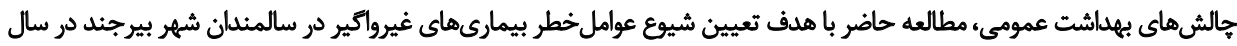

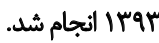

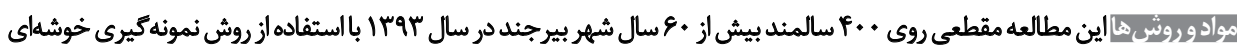

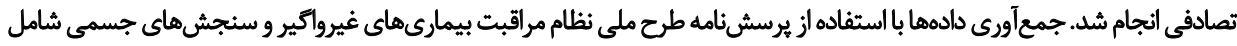

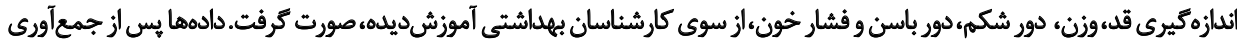

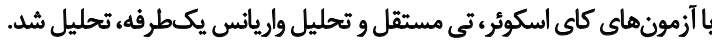

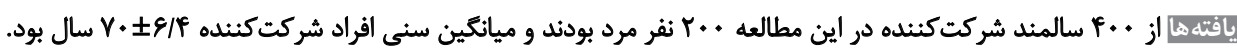

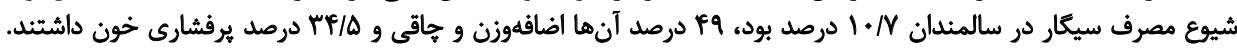

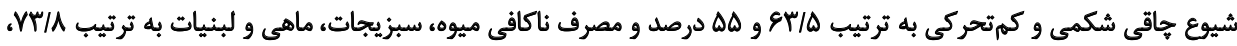

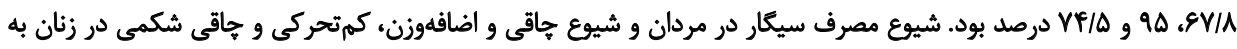

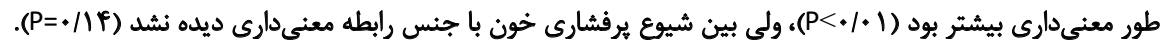

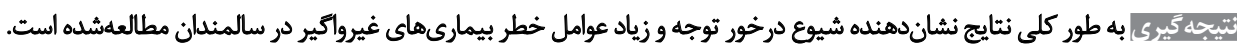

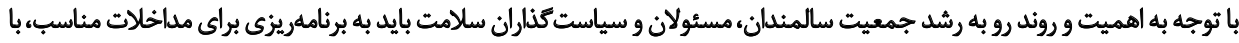

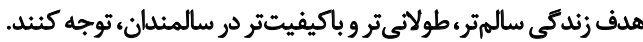

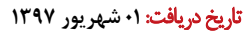

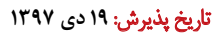

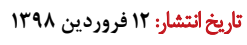

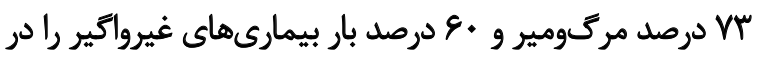

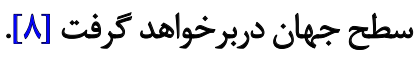

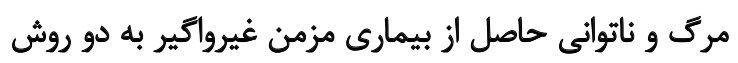

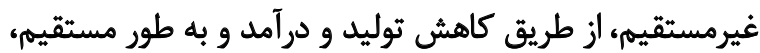

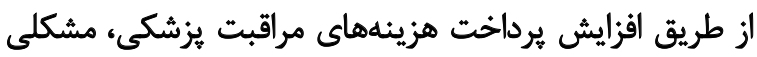

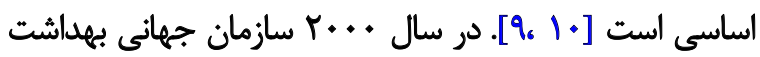

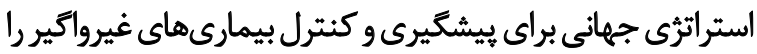

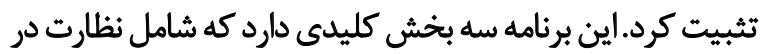

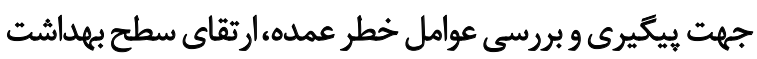

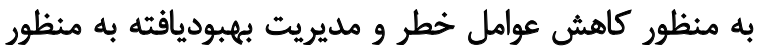
دسترسى به خدمات مراقبت سلامت است [ماير [1].
مقدمه

بيمارىهاى مزمن غيرواكير، مشكل بهداشت جهانى و تهديدى

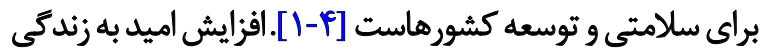

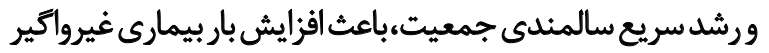

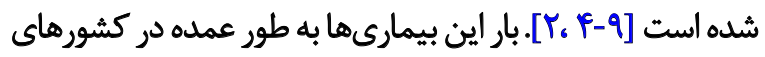

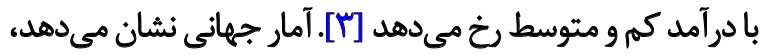

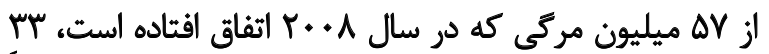

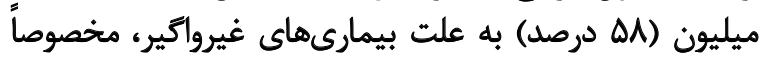

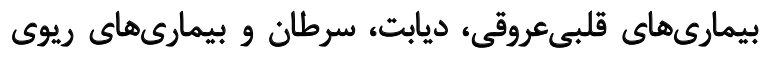

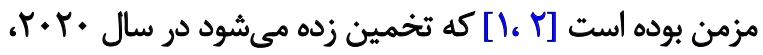

ㅁ.

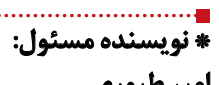

المير طيورى نوينده مئر نشائى: تهران، دانشكاه علوم يزشكى و خدمات بههاشتى درمانى ايران، دانشكده بهداشت، كروه إبيدميولوزى. ت تلفن: amir.turi@yahoo.com تيست الكترونيكي: 


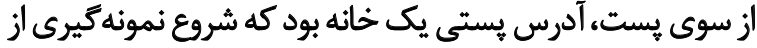

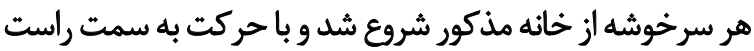

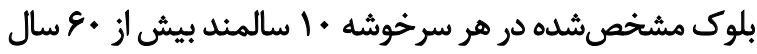
شامل ه مرد و ه زنَ، وارد مطالعه شدرند.

قبل از يركردن يرسشنامهها، اهداف مطالعه براي سالمندان

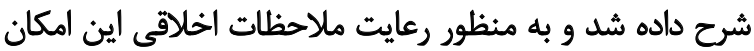

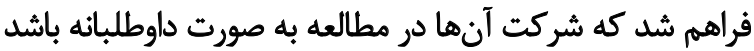

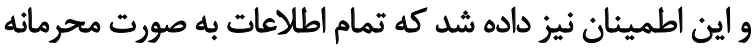
نزد محقق باقي خواهد ماند.

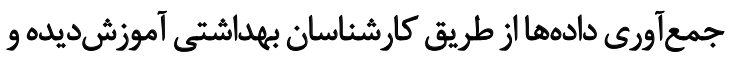

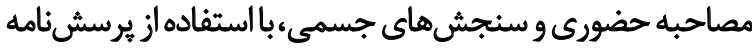

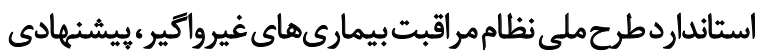

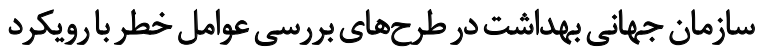

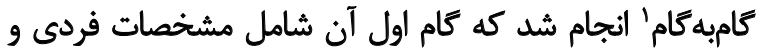

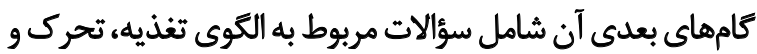

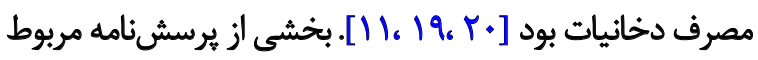

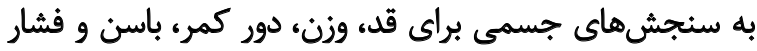

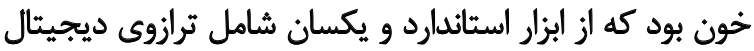

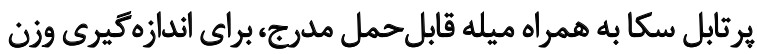

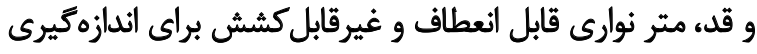

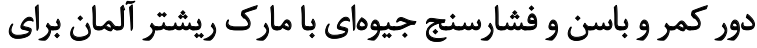
اندازهيرى فشار خون استفاده شد.

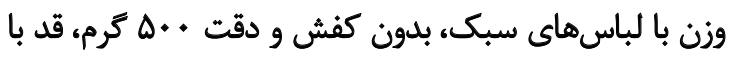

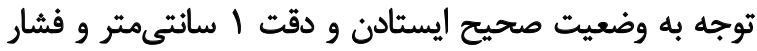

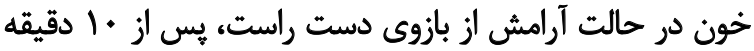

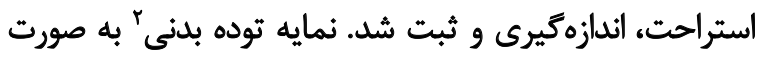

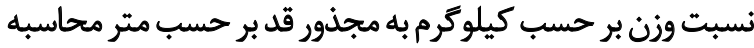

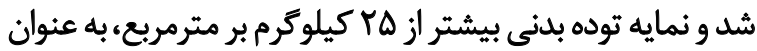

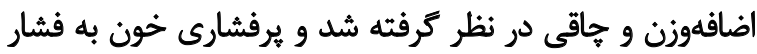

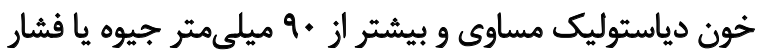

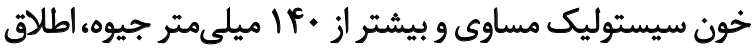

تبراى اندازهيرى دور كمر تقريباً نقطه وسط فاصله بين حاشيه

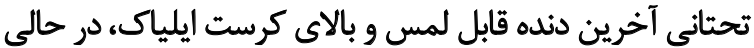

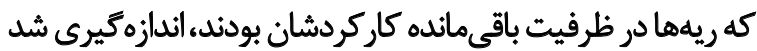

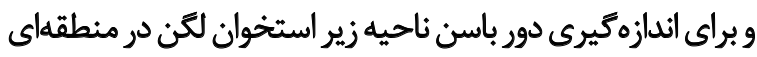

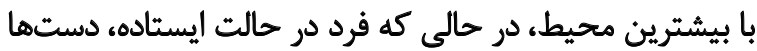

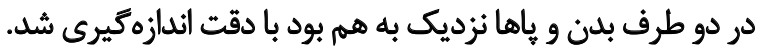

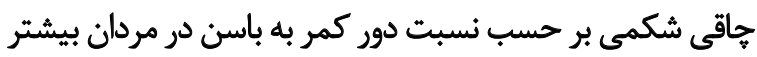

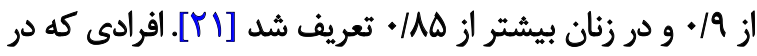

1. Who steps instrument

2. Body Mass Index (BMI)
عوامل خطر عمده و اصلى بيمارىهاى مزمن غيرواكير در

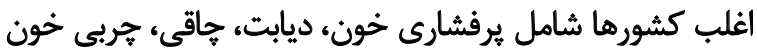

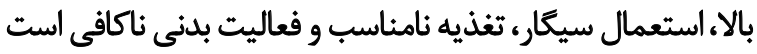

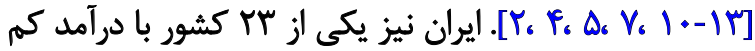

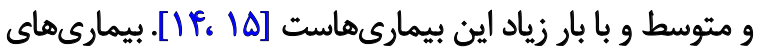

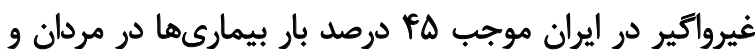

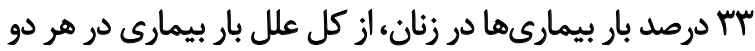

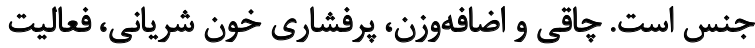

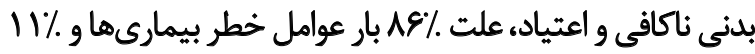

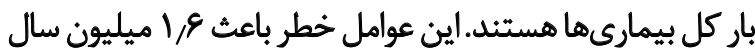

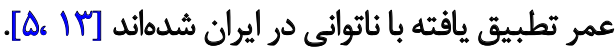
بر اساس مطالعه ملى انجامشده روى سالمندان ايرانى در دري

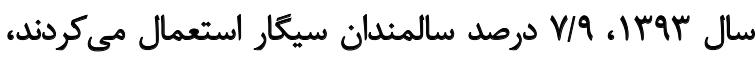

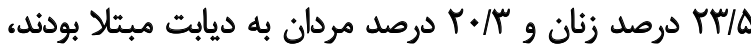

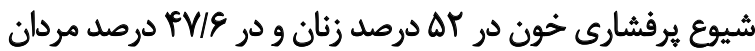

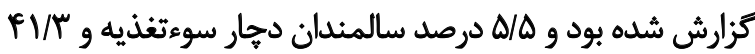

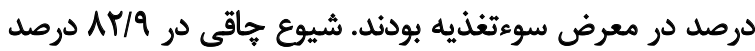

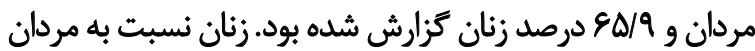

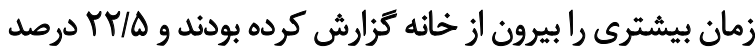

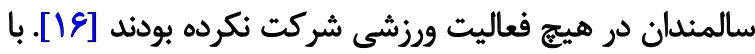

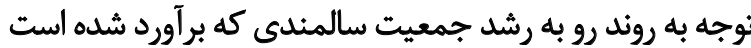

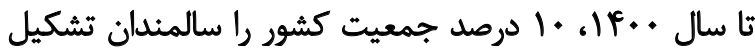

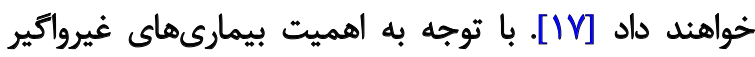

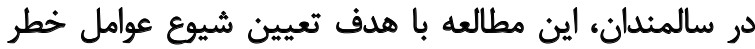

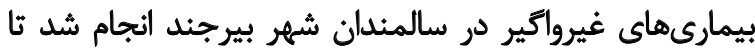

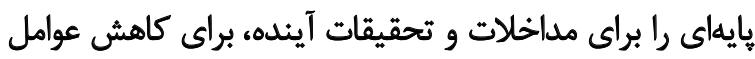

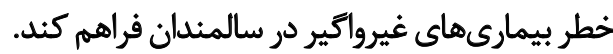
روش مطالعه

ايرؤهش حاضر از نوع مقطعى (توصيفى تحليلى) بود كه جامعه

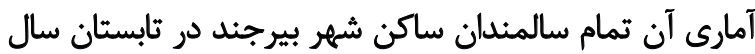

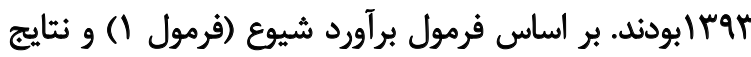

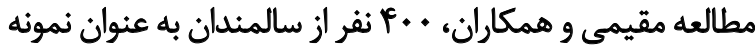

به روش خوشهاى تصادفى انتخاب شدند [1/1] فرمول ا. حجم نمونه براى برآورد شيوع

$$
n=\frac{z^{2} \times p q}{d^{2}}
$$

ابتدا شهر بيرجند بر اساس وضعيت جغرافيايى به f منطقيه

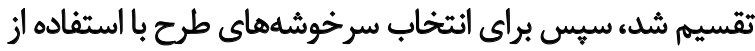

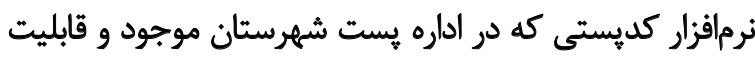

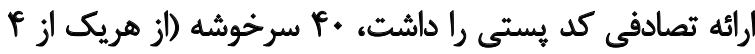

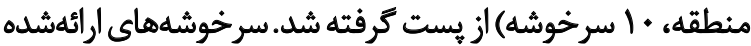




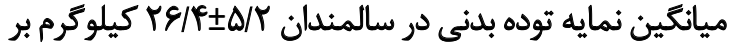

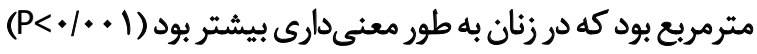

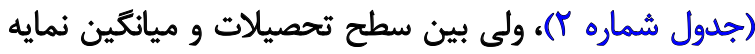

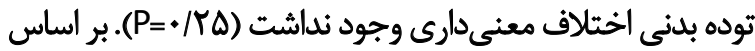

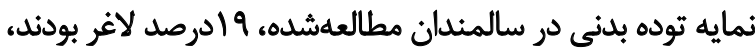

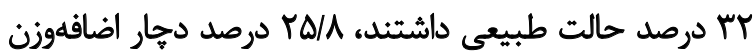

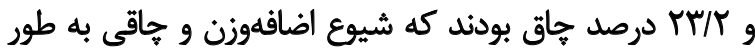

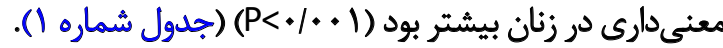

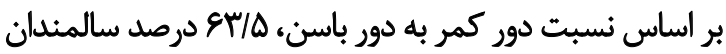

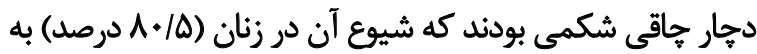

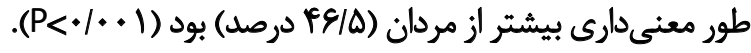
ميانكين فشارخون سيستوليك و دياستوليك سالمندان

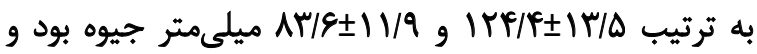

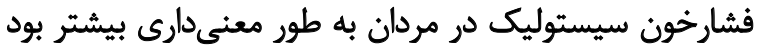

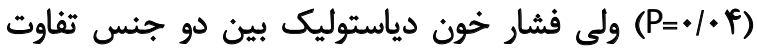

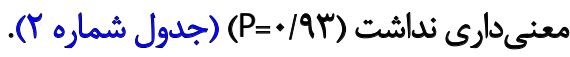

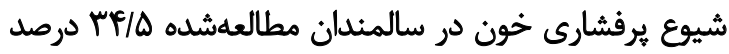

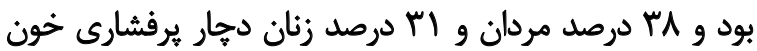

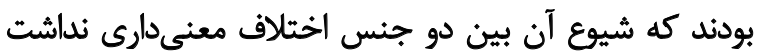

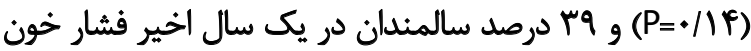
خود را اندازميرى نكرده بودند.

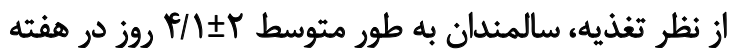

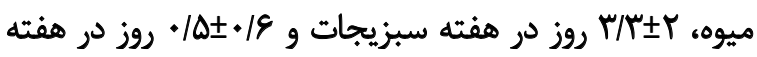

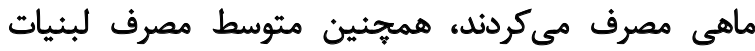

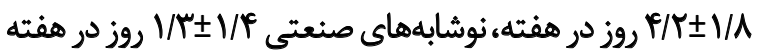

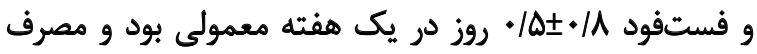

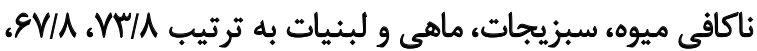

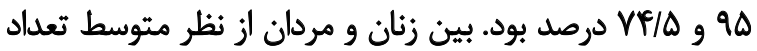

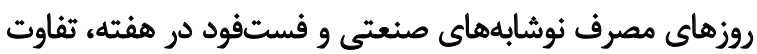

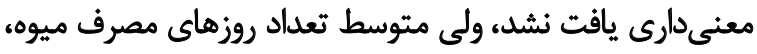

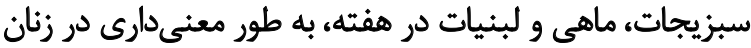

زمان مطالعهء، روزانه حداقل يك نخ سيكار مصرف مي كردند، به

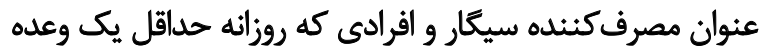

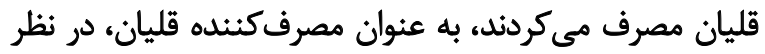

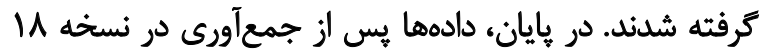

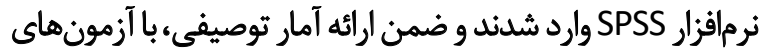

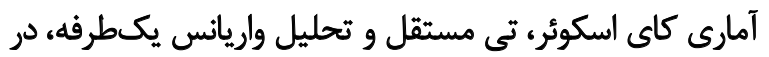

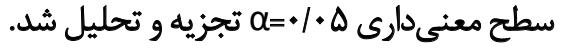

ياقتهنها

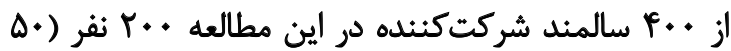

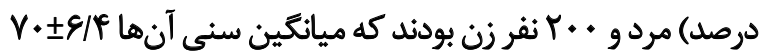

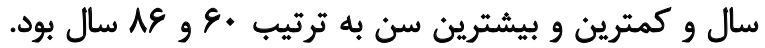

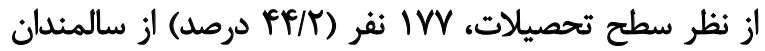

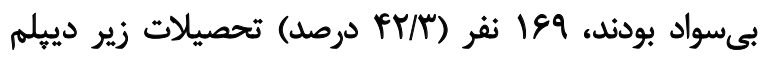

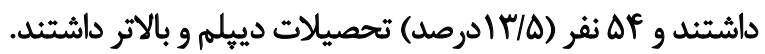

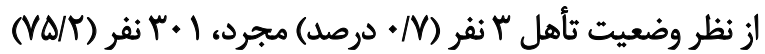

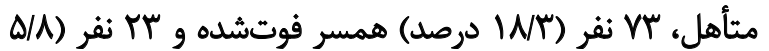
درصد) طلاق ترفته از همسر بودند.

از نظر روغن مصرفى در خانوار س/ربه درصد سالمندان روغن

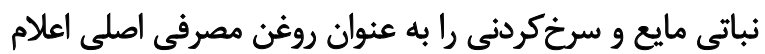

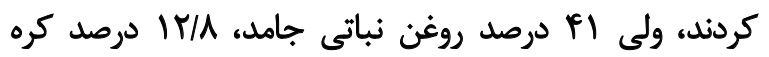

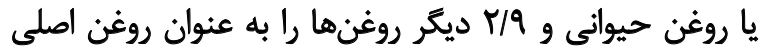

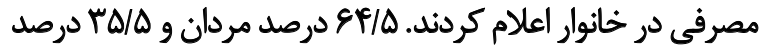

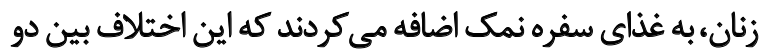

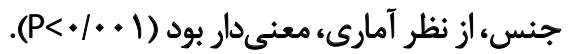

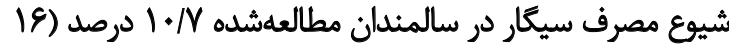

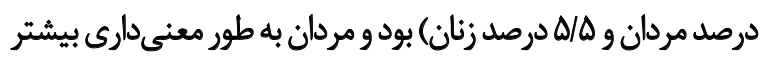

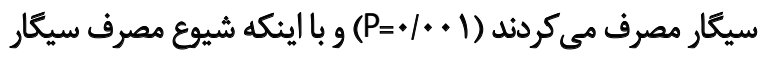

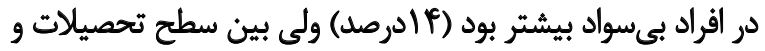

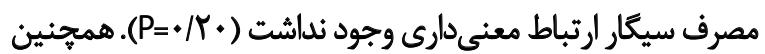

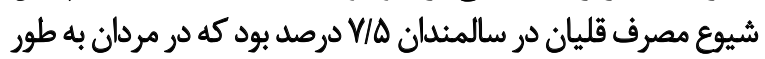

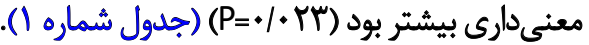

جدول ا. مقايسه شيوع مصرف سيكار، قليان، اضافهوزن و جاقى و كمتحركى بر حسب جنسيت در سالمندان مطالعهشده

\begin{tabular}{|c|c|c|c|c|}
\hline \multicolumn{4}{|c|}{ تعداد (درصد) } & \multirow{2}{*}{ جنسيت } \\
\hline شيوع كمتحركى & شيوع اضافهوزن و هاقى & شيوع مصرف قليان & شيوع مصرف سيكار & \\
\hline$q(f \wedge)$ & $V \in(m)$ & $r(1+/ \Delta)$ & $M(18)$ & 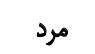 \\
\hline $\operatorname{Irf}(g T)$ & $\operatorname{Irr}(8)$ & $q(Y / \Delta)$ & $\Pi(\Delta / \Delta)$ & ن \\
\hline $1+\infty$ & $<+1+\infty 1$ & TH & $.1++1$ & $\mathbf{P}$ \\
\hline$M \cdot(\Delta \Delta)$ & $198(199)$ & $r \cdot(V / \Delta)$ & $R(1 . / N)$ & جمع \\
\hline
\end{tabular}

证 
جدول r. مقايسه ميانكين نمايه توده بدنى و فشار خون دياستوليك و سيستوليك برحسب جنسيت در سالمئدان مطالعهشده

\begin{tabular}{|c|c|c|c|}
\hline \multicolumn{3}{|c|}{ ميانكين+|نحراف معيار } & \multirow{2}{*}{ جنسيت } \\
\hline نمايه توده بدنى & فشار خُون سيستوليك & فُشار خون دياستوليك & \\
\hline$r f / a \pm r / q$ & $|r \Delta / A \pm| r / \mid$ & $\Lambda H / \Delta \pm I r / N$ & 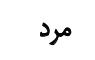 \\
\hline$r V / \Lambda \pm \Delta / q$ & $\mid r \Psi \pm I T / Y$ & $A r / 8 \pm 11$ & زن - - ت - ت \\
\hline$<\cdot 1 \cdot .1$ & $.1 . p^{f}$ &.$/ 94$ & $\mathrm{P}$ \\
\hline
\end{tabular}

ill

[TM]. در مطالعات روى سالمندان ديكّر مناطق جهان مطالعه

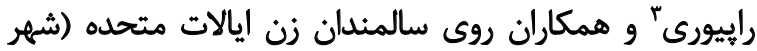

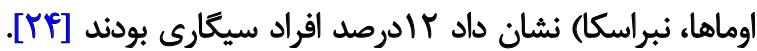

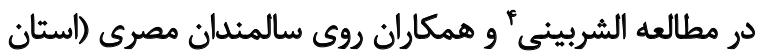

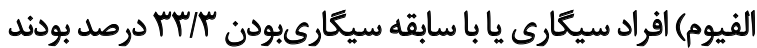

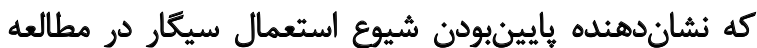

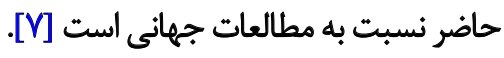

با توجه به نتايج، مصرف دخانيات در سالمندان مطالعهشده

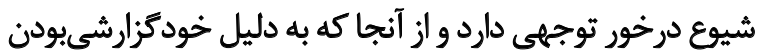

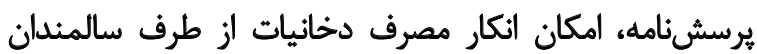

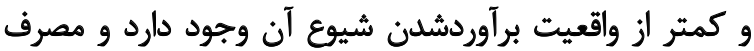

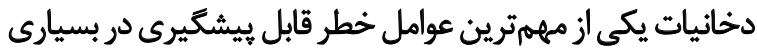

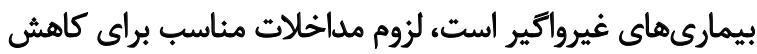

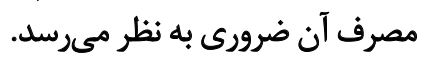

بر اساس نتايج، ميانظين فشار خون سيستوليك و دياستوليك

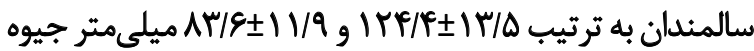

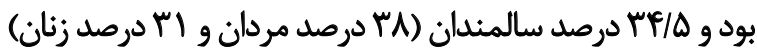

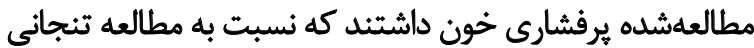

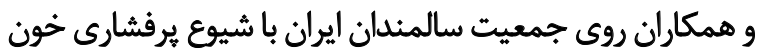

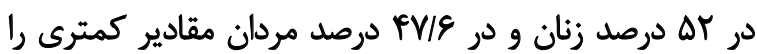

\section{Rapuri}

4. El-Sherbiny

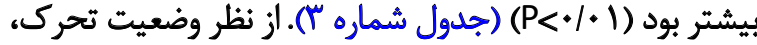

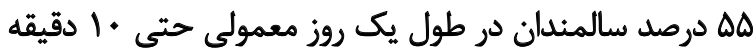

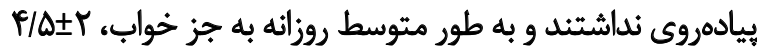

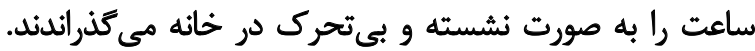

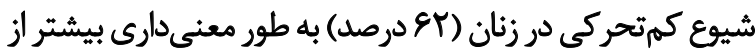

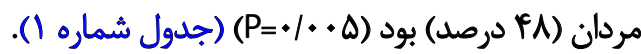

ب

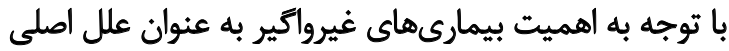

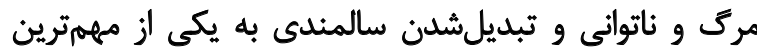

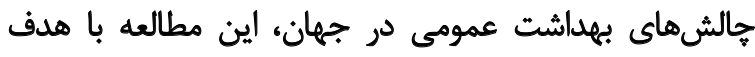

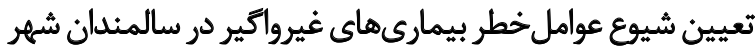

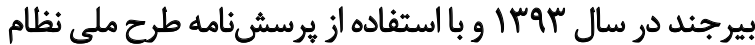
مراقبت بيمارى هاى غيرواكير انجام شد.

نتايج مطالعه حاضر نشان داد، شيوع استعمال سيعار در دردار

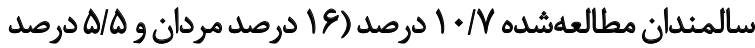

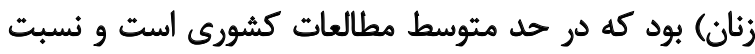

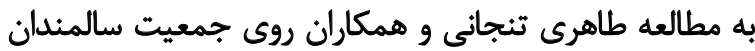

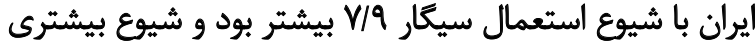

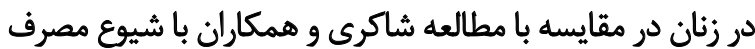

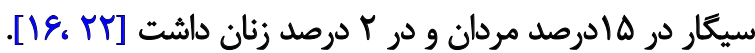

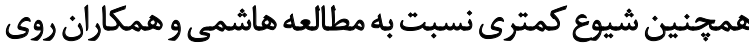

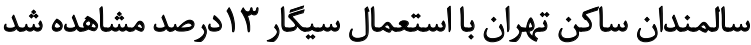

جدول بـ. مقايسه ميانكين تعداد روزهاى مصرف ميوه، سبزيجات، ماهي و لبنيات در هفته برحسب جنسيت در سالمندان مطالعهشده

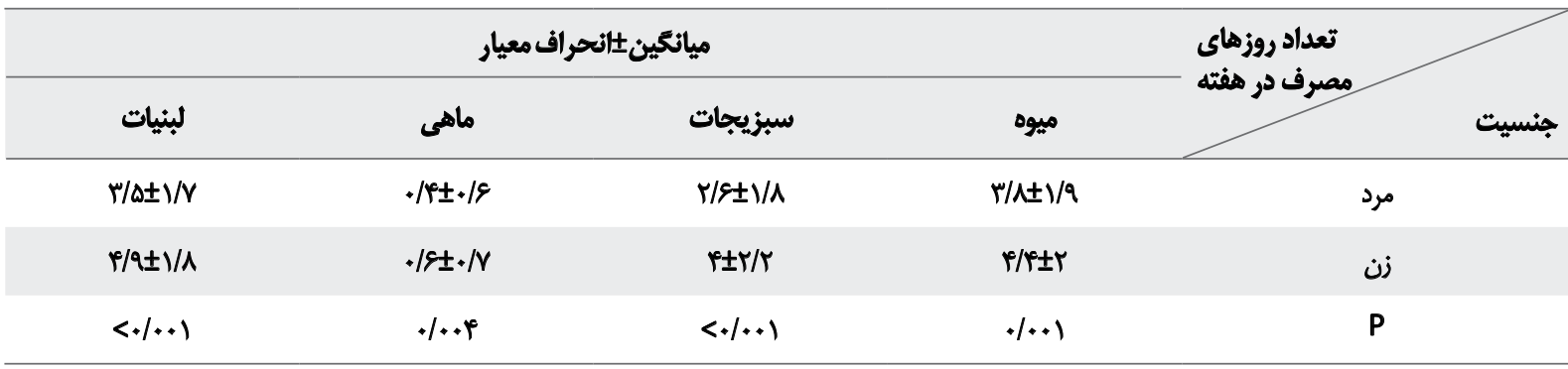


نتايج درباره تغذيه سالمندان نشان داد از نظر تغذيه، سالمندان

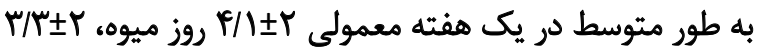

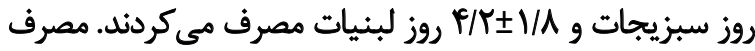

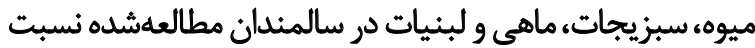

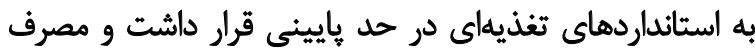

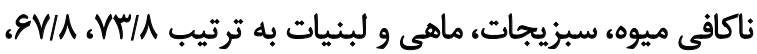

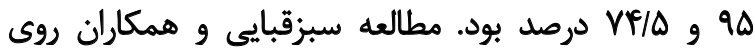

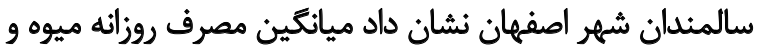

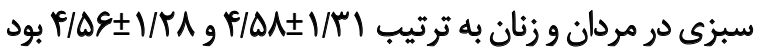

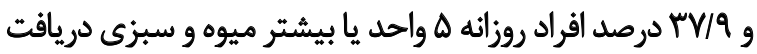

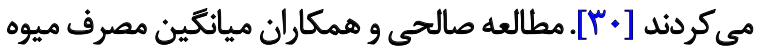

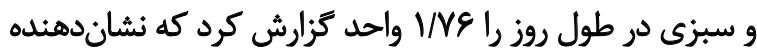

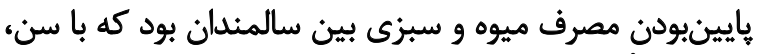

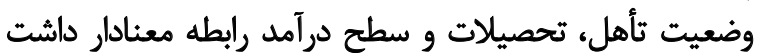

در مطالعات روى سالمثدان ديكر نقاط جهان، مطالعه نوامارال

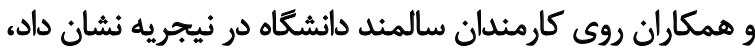

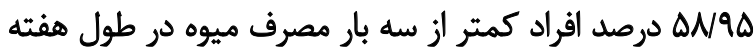

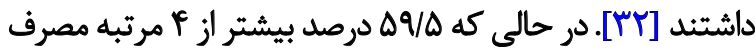

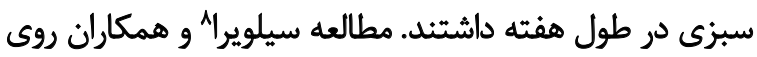

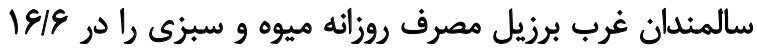

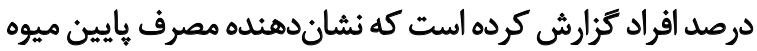

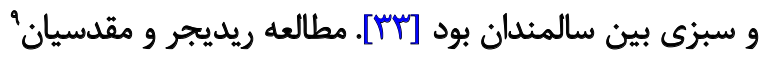

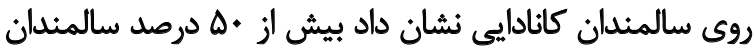

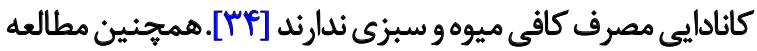

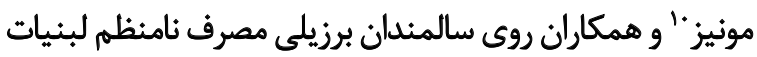

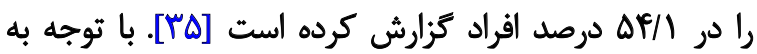

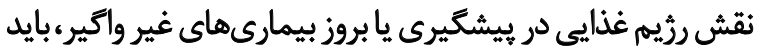

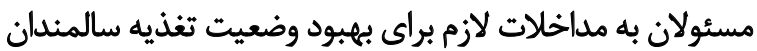

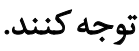

بر اساس نتايج مطالعه حاضر ه ه درصد سالمندان مطالعهشده دجار كمتحركى بودند. مطالعه اسحاقى و همكاراران، مطالعه

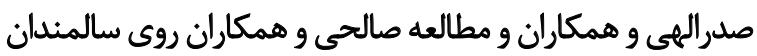

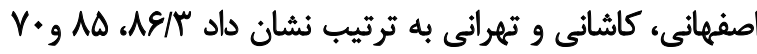

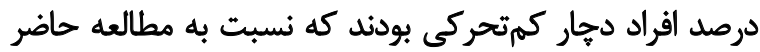

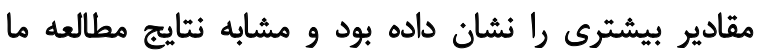

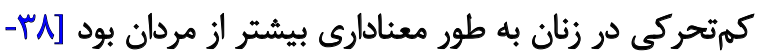

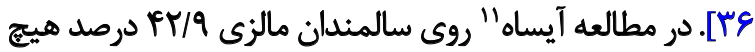

7. Nwamarah

8. Silveira

9. Riediger \& Moghadasian

10. Muniz

11. Ayiesah
نشان داد و شيوع كمترى در مقايسه با شيوع يرفشارى خون

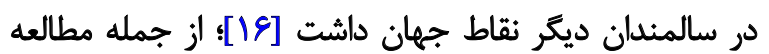

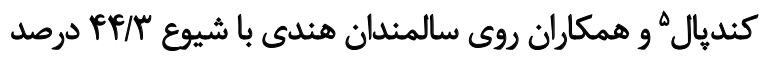

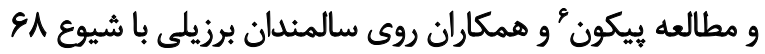

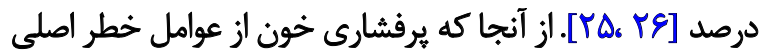

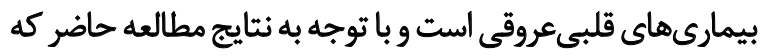

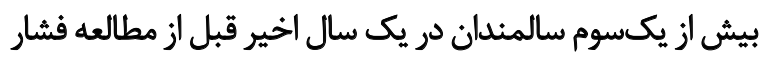

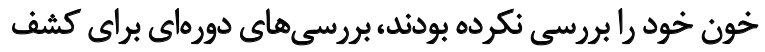

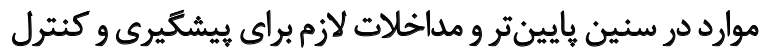
مناسب آن ضرورى به نظر مير مئد

ميانكين نمايه توده بدنى در سالمندان مطالعشده

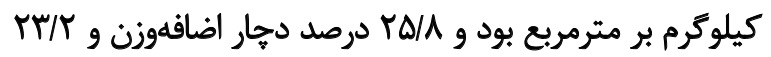

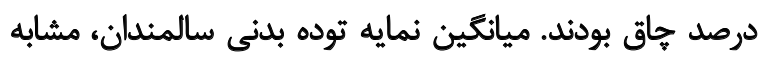

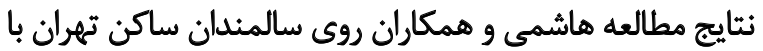

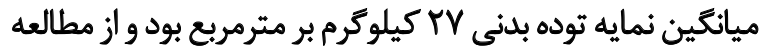

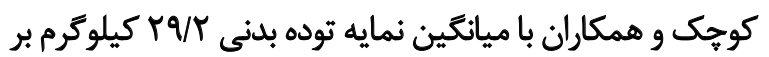

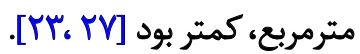

اين مطالعه، در شيوع هاقى، نسبت به مطالعه تنجانى و و دوراقي

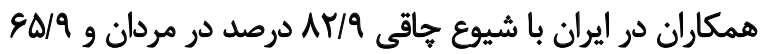

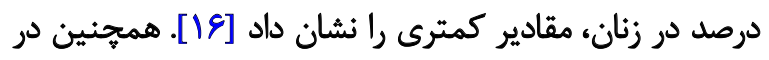

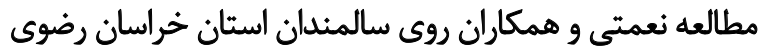

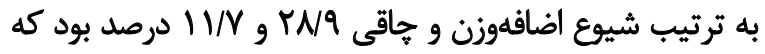

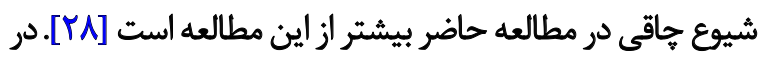

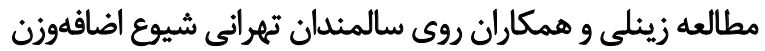

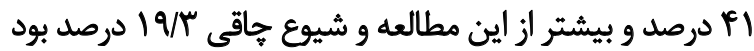

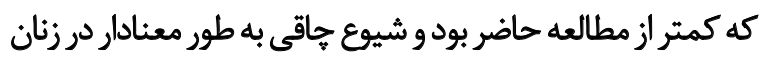

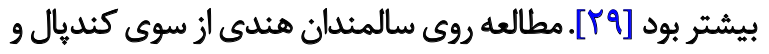

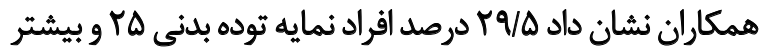

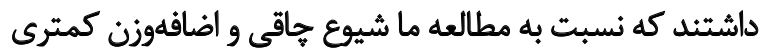

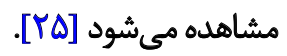

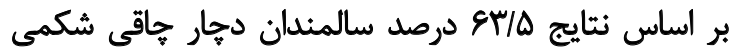

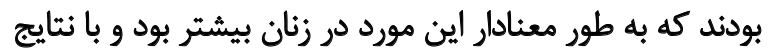

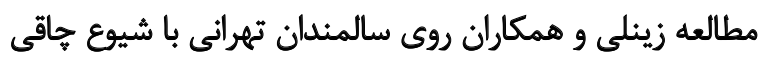

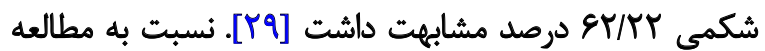

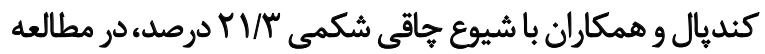

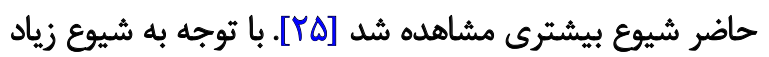

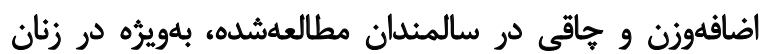

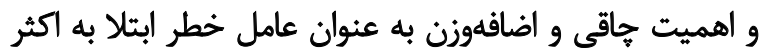

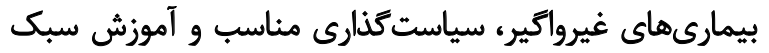

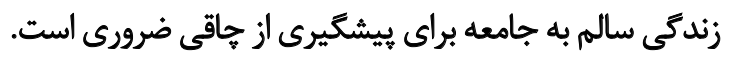

5. Kandpal

6. Picon 


$$
\text { تشكر و قدردانى }
$$

بدينوسيله از معاونت تحقيقات و فناورى دانشكاه علوم يزشكى بيرجند تقدير و تشكر مي كنيم.
شركتى در فعاليت فيزيكى نداشتند كه مشابه نتايج مطالعه

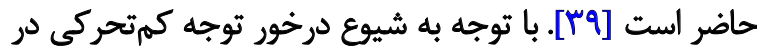

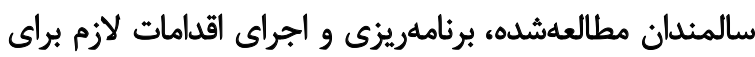

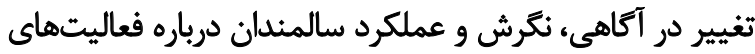
ورزشى ضرورى به نظر مىرسد.

\section{نتيجلئيرى نهايى}

به طور كلى نتايج اين مطالعه نشاندهنده شيوع درخور توجه و

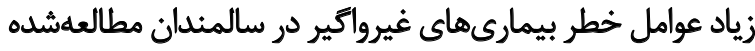

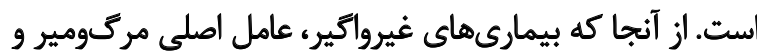

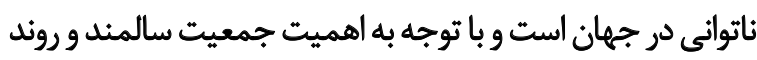

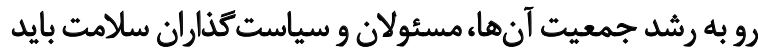

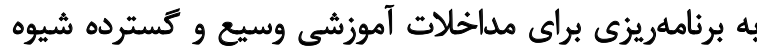

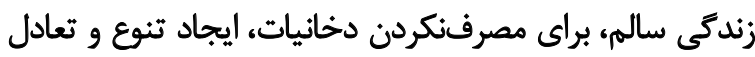

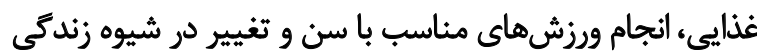

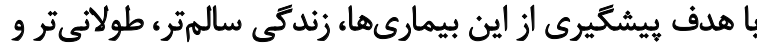
باكيفيتر در سالمندان، توجه كنيند.

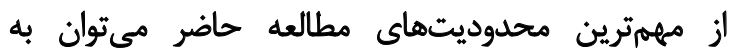

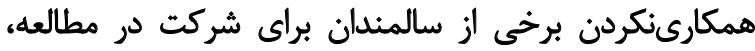

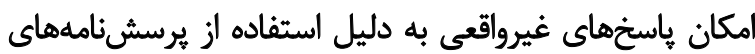

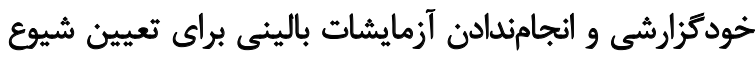

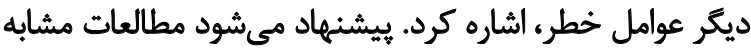

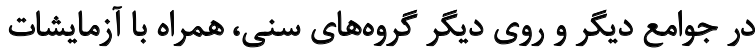

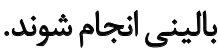
مالاحظات اخلاقي بيروى از اصول اخلاق يثوهش

كميته اخلاق دانشعاه علوم يزشكى بيرجند مطالعه حاضر رابا كد IR.BUMS.1394.82 تصويب كرده است.

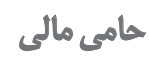

اين مقاله حاصل طرح تحقيثاتى دانشجويى مصوب دانشَّاه

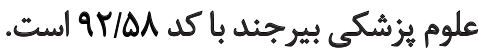

$$
\text { مشاركت نويسند مكان }
$$

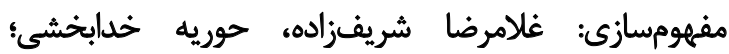

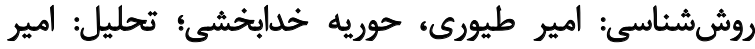

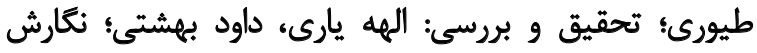

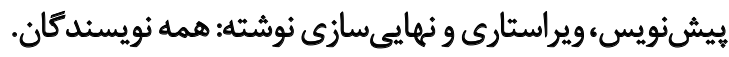

$$
\text { تعارض منافع }
$$

بنابر اظهار نويسندكان، اين مقاله تعارض منافع ندارد. 


\section{References}

[1] Alwan A, MacLean DR, Riley LM, d'Espaignet ET, Mathers CD, Stevens GA, et al. Monitoring and surveillance of chronic noncommunicable diseases: Progress and capacity in high-burden countries. The Lancet. 2010; 376(9755):1861-8. [DOI:10.1016/ S0140-6736(10)61853-3]

[2] Boutayeb A, Boutayeb S, Boutayeb W. Multi-morbidity of non communicable diseases and equity in WHO Eastern Mediterranean countries. International Journal for Equity in Health. 2013; 12(60):2-8. [DOI:10.1186/1475-9276-12-60] [PMID] [PMCID]

[3] Goeppel C, Frenz P, Tinnemann P, Grabenhenrich L. Universal health coverage for elderly people with non-communicable diseases in low-income and middle-income countries: A crosssectional analysis. The Lancet. 2014; 384:S6. [DOI:10.1016/S01406736(14)61869-9]

[4] Li Q, Guo J, Cao XQ, Yuan X, Rao KQ, Zheng Z, et al. Trend of non-communicable disease mortality for three common conditions in the elderly population from 2002 to 2010: A populationbased study in China. Chronic Diseases and Translational Medicine. 2015; 1(3):152-7. [DOI:10.1016/j.cdtm.2015.06.006] [PMID] [PMCID]

[5] Asgari F, Aghajani H, Haghazali M, Heidarian H. Non-communicable diseases risk factors surveillance in Iran. Iranian Journal of Public Health. 2009; 38(Supple 1):119-22.

[6] Corrêa MM, Thumé E, De Oliveira ERA, Tomasi E. Performance of the waist-to-height ratio in identifying obesity and predicting non-communicable diseases in the elderly population: A systematic literature review. Archives of Gerontology and Geriatrics. 2016; 65:174-82. [DOI:10.1016/j.archger.2016.03.021] [PMID]

[7] El-Sherbiny NA, Younis A, Masoud M. A comprehensive assessment of the physical, nutritional, and psychological health status of the elderly populace in the Fayoum Governorate (Egypt). Archives of Gerontology and Geriatrics. 2016; 66:119-26. [DOI:10.1016/j.archger.2016.06.001] [PMID]

[8] Krishnan A, Shah B, Lal V, Shukla D, Paul E, Kapoor S. Prevalence of risk factors for non-communicable disease in a rural area of Faridabad district of Haryana. Indian Journal of Public Health. 2008; 52(3):117-24. [PMID]

[9] Muka T, Imo D, Jaspers L, Colpani V, Chaker L, van der Lee SJ, et al. The global impact of non-communicable diseases on healthcare spending and national income: A systematic review. European Journal of Epidemiology. 2015; 30(4):251-77. [DOI:10.1007/ s10654-014-9984-2] [PMID]

[10] Dans A, Ng N, Varghese C, Tai ES, Firestone R, Bonita R. The rise of chronic non-communicable diseases in southeast Asia: Time for action. The Lancet. 2011; 377(9766):680-9. [DOI:10.1016/ S0140-6736(10)61506-1]

[11] Esteghamati A, Khalilzadeh O, Rashidi A, Meysamie A, Haghazali M, Asgari F, et al. Association between physical activity and insulin resistance in Iranian adults: National Surveillance of Risk Factors of Non-Communicable Diseases (SuRFNCD-2007). Preventive Medicine. 2009; 49(5):402-6. [DOI:10.1016/j.
[ ypmed.2009.09.005] [PMID]

[12] Mayosi BM, Flisher AJ, Lalloo UG, Sitas F, Tollman SM, Bradshaw D. The burden of non-communicable diseases in South Africa. The Lancet. 2009; 374(9693):934-47. [DOI:10.1016/S01406736(09)61087-4]
[13] Sarrafzadegan N, Baghaei A, Sadri G, Kelishadi R, Malekafzali $\mathrm{H}$, Boshtam M, et al. Isfahan healthy heart program: Evaluation of comprehensive, community-based interventions for noncommunicable disease prevention. Prevention and Control. 2006; 2(2):73-84. [DOI:10.1016/j.precon.2006.10.003]

[14] Habibzadeh F. The control of non-communicable diseases in rural Iran. The Lancet. 2012; 379(9810):6-7. [DOI:10.1016/S01406736(11)61678-4]

[15] Kilpi F, Webber L, Musaigner A, Aitsi-Selmi A, Marsh T, Rtveladze K, et al. Alarming predictions for obesity and non-communicable diseases in the Middle East. Public Health Nutrition. 2014; 17(5):1078-86. [DOI:10.1017/S1368980013000840] [PMID]

[16] Tanjani PT, Motlagh ME, Nazar MM, Najafi F. The health status of the elderly population of Iran in 2012. Archives of Gerontology and Geriatrics. 2015; 60(2):281-7. [DOI:10.1016/j.archger.2015.01.004] [PMID]

[17] Fotoukian Z, Mohammadi Shahboulaghi F, Fallahi Khoshknab M. [Analytical on empowerment interventions in older people with chronic disease: A review literature (Persian)]. Journal of Health Promotion Management. 2013; 2(4):65-76.

[18] Moghimi M, Salari M, Abasi A, Saniyi F, Nemati S. [Prevalence of hypertension and its risk factors in elderly Yasuj 2005 (Persian)]. Dena, Journal of Nursing and Midwifery. 2006; 1(2):61-70.

[19] Khademi N, Gooya MM, Izade B, Mansory F, Laghai Z. [Decreasing risk factors of non-communicable diseases among health workers of Kermanshah university of medical sciences (20082010) (Persian)]. Hakim Research Journal. 2013; 15(4):330-8.

[20] Nasehi M, Moosazadeh M, Amiresmaeili M, Parsaee M, Zakizadeh R, Mirzajani M. [Prevalence of five main risk factors of non-communicable diseases in Mazandaran Province: A population based study (Persian)]. Journal of Mazandaran University of Medical Sciences. 2012; 21(86):193-202.

[21] World Health Organization. Waist circumference and waisthip ratio: Report of a WHO expert consultation, Geneva, 8-11 December 2008. Geneva: World Health Organization; 2011.

[22] Shakeri N, Eskandari F, Hajsheikholeslami F, Ghanbarian A, Azizi F. On the estimation of survival time and contributory risk factors in the elderly participants in the Tehran lipid and glucose study. A 10-year follow-up. Journal of Paramedical Sciences. 2011; 2(2):2-8. [DOI:10.22037/jps.v2i2.2349]

[23] Hashemi R, Motlagh AD, Heshmat R, Esmaillzadeh A, Payab $\mathrm{M}$, Yousefinia $\mathrm{M}$, et al. Diet and its relationship to sarcopenia in community dwelling Iranian elderly: A cross sectional study. Nutrition. 2015; 31(1):97-104. [DOI:10.1016/j.nut.2014.05.003] [PMID]

[24] Rapuri PB, Gallagher JC, Smith LM. Smoking is a risk factor for decreased physical performance in elderly women. The Journals of Gerontology Series A: Biological Sciences and Medical Sciences. 2007; 62(1):93-9. [DOI:10.1093/gerona/62.1.93]

[25] Kandpal S, Kakkar R, Aggarwal P, Bansal S. Pattern of prevalence of risk factors for non-communicable diseases in the geriatric population of district Dehradun. Journal, Indian Academy of Clinical Medicine. 2013; 14(3-4):214-7.

[26] Picon RV, Fuchs FD, Moreira LB, Fuchs SC. Prevalence of hypertension among elderly persons in urban Brazil: A systematic review with meta-analysis. American Journal of Hypertension. 2013; 26(4):541-8. [DOI:10.1093/ajh/hps076] [PMID] 
[27] Koochek A, Johansson S, Kocturk T, Sundquist J, Sundquist K. Physical activity and body mass index in elderly Iranians in Sweden: A population-based study. European Journal of Clinical Nutrition. 2008; 62(11):1326-32. [DOI:10.1038/sj.ejcn.1602851] [PMID]

[28] Nematy M, Sakhdari A, Ahmadi-Moghaddam P, Aliabadi M, Kimiagar M, llaty A, et al. Prevalence of obesity and its association with socioeconomic factors in elderly Iranians from RazaviKhorasan province. The Scientific World Journal. 2009; 9:1286-93. [DOI:10.1100/tsw.2009.139] [PMID] [PMCID]

[29] Zeinali F, Samadi M, Azam K, Djafarian K. [Body composition among elderly and its relationship with physical activity pattern (Persian)]. Journal of Mazandaran University of Medical Sciences. 2016; 26(135):62-74.

[30] Sabzghabaee AM, Mirmoghtadaee P, Mohammadi M. Fruit and vegetable consumption among community dwelling elderly in an Iranian population. International Journal of Preventive Medicine. 2010; 1(2):98-102. [PMID] [PMCID]

[31] Salehi LEH, Mohammad K, TavafianSS, Jazayery A, Montazeri A. Consumption of fruit and vegetables among elderly people: a cross sectional study from Iran. Nutrition Journal. 2010; 9(1):1. [DOI:10.1186/1475-2891-9-2] [PMID] [PMCID]

[32] Nwamarah JU, Otitoju GTO. Fruit and vegetable consumption pattern and health challenges of elderly ( $\geq 60$ Years) staff in the University of Nigeria, Nsukka and Enugu Campuses: A case study. Pakistan Journal of Nutrition. 2014; 13(11):626 [DOI:10.3923/pjn.2014.626.630]

[33] Silveira EA, Martins BB, Abreu LRSd, Cardoso CKdS. Low consumption of fruit, vegetables and greens: associated factors among the elderly in a Midwest Brazilian city. Ciência \& Saúde Coletiva. 2015; 20(12):3689-99. [DOI:10.1590/1413-812320152012.07352015] [PMID]

[34] Riediger ND, Moghadasian MH. Patterns of fruit and vegetable consumption and the influence of sex, age and socio-demographic factors among Canadian elderly. Journal of the American College of Nutrition. 2008; 27(2):306-13. [DOI:10.1080/07315724.200 8.10719704]

[35] Muniz LC, Madruga SW, Araújo CL. Consumption of dairy products by adults and the elderly in the south of Brazil: A population-based study. Ciência \& Saúde Coletiva. 2013; 18(12):351522. [DOI:10.1590/S1413-81232013001200008]

[36] Eshaghi SR, Shahsanai A, Ardakani MM. [Assessment of the physical activity of elderly population of Isfahan, Iran (Persian)]. Journal of Isfahan Medical School. 2011; 29(147):939-46.

[37] Sadrollahi A, Hosseinian M, Alavi NM, Khalili Z, Esalatmanesh S. Physical activity patterns in the elderly Kashan population. Iranian Red Crescent Medical Journal. 2016; 18(6):e25008. [DOI:10.5812/ircmj.25008]

[38] Salehi L, Eftekhar H, Mohammad K, Taghdisi MH, Shojaeizadeh D. Physical activity among a sample of Iranians aged over 60 years: an application of the transtheoretical model. Archives of Iranian Medicine. 2010; 13(6):528-36.

[39] Ayiesah R. The level of physical activities amongst elderly in a community. Journal of Health and Translational Medicine (JUMMEC). 2007; 10(1):29-33. [DOI:10.22452/jummec.vol10no1.7] 
This Page Intentionally Left Blank 